\title{
Increasing the precision of orthology-based complex prediction through network alignment
}

Macromolecular assemblies play an important role in almost all cellular processes. However, despite several large-scale studies, our current knowledge about protein complexes is still quite limited, thus advocating the use of in silico predictions to gather information on complex composition in model organisms. Since protein-protein interactions present certain constraints on the functional divergence of macromolecular assemblies during evolution, it is possible to predict complexes based on orthology data. Here, we show that incorporating interaction information through network alignment significantly increases the precision of orthology-based complex prediction. Moreover, we performed a large-scale in silico screen for protein complexes in human, yeast and fly, through the alignment of hundreds of known complexes to whole organism interactomes. Systematic comparison of the resulting network alignments to all complexes currently known in those species revealed many conserved complexes, as well as several novel complex components. In addition to validating our predictions using orthogonal data, we were able to assign specific functional roles to the predicted complexes. In several cases, the incorporation of interaction data through network alignment allowed to distinguish real complex components from other orthologous proteins. Our analyses indicate that current knowledge of yeast protein complexes exceeds that in other organisms and that predicting complexes in fly based on human and yeast data is complementary rather than redundant. Lastly, assessing the conservation of protein complexes of the human pathogen Mycoplasma pneumoniae, we discovered that its complexes repertoire is different from that of eukaryotes, suggesting new points of therapeutic intervention, whereas targeting the pathogen's Restriction enzyme complex might lead to adverse effects due to its similarity to ATP-dependent metalloproteases in the human host. 
1 List of authors:

2 Roland A. Pache ${ }^{1,2, *}$ and Patrick Aloy ${ }^{1,3}$

3 1. Joint IRB-BSC Program in Computational Biology, Institute for Research in

4 Biomedicine (IRB Barcelona), c/ Baldiri Reixac 10-12, 08028 Barcelona, Spain

5 2. Current affiliation: Department of Bioengineering and Therapeutic Sciences,

6 University of California San Francisco (UCSF), $17004^{\text {th }}$ street, 94158 San Francisco,

7 California, USA

8 3. Institució Catalana de Recerca i Estudis Avançats (ICREA), Passeig Lluís Companys

9 23, 08010 Barcelona, Spain

$10{ }^{*}$ Corresponding author:

11 Roland A. Pache. Tel: +1 415514 4307; Email: roland.pache@ucsf.edu

12 Email addresses:

13 RAP: roland.pache@ucsf.edu

14 PA: patrick.aloy@irbbarcelona.org 


\section{Introduction}

Almost every major process in a cell, such as replication, transcription, translation and degradation, is carried out not by single proteins, but by macromolecular complexes, regulated through intricate networks of protein-protein interactions. To understand cellular behaviour on a systemic level, we thus need a comprehensive knowledge of the protein complexes present in the respective organism.

In the last years, many small-scale studies have identified protein complexes in yeast and human, which have been collected in the public databases MPACT [1] and CORUM [2], respectively. Moreover, several large-scale proteomics experiments, using tandemaffinity purification coupled to mass spectrometry analysis, have focused on systematically unveiling the composition of macromolecular complexes in the budding yeast Saccharomyces cerevisiae $[\underline{3}, \underline{4}, \underline{5}, \underline{6}, \underline{7}]$, the human pathogen Mycoplasma pneumoniae [8], and recently also in the fruit fly Drosophila melanogaster [9] and in human $[10,11]$. Although yeast is the least complex eukaryotic model organism with about 6,000 genes, several hundreds of protein complexes were found, and the total number of complexes in yeast was estimated to be over 800 []ㅡ. Moreover, the first screen in M. pneumonia already yielded 62 homo- and 116 heteromultimeric protein complexes, despite the pathogen's small genome of only 689 protein-coding genes [8]. The importance of protein complexes for cell survival becomes apparent when probing the essentiality of their protein-coding genes through knock-out mutations. Indeed, several studies have shown that protein complexes in yeast are significantly enriched in essential genes $[12,13,14,15]$. To discover the molecular details of how individual proteins function together as macromolecular assemblies, follow-up initiatives have then aimed at identifying those complexes that are suitable for structural studies by combining systematic bioinformatics and experimental validation strategies [16,17].

So far, however, these important investigations, which have improved our understanding of the architecture and function of protein complexes, were limited to yeast, not only due to the scarcity of functional data in other organisms, but also because their protein complexes are yet largely unknown. To determine whether the findings for yeast complexes describe general principles of molecular organization and to discover how protein complexes have evolved, it is thus necessary to define protein complexes in other species, complementing the results of recent screening efforts $[\underline{9}, \underline{10}, 11]$. Since the 
experimental characterization of macromolecular assemblies is difficult and requires large amounts of time and resources, predicting protein complexes based on existing protein-protein interaction (PPI) and orthology data becomes an interesting alternative. Indeed, different strategies have been developed to exploit these data. On the one hand, several graph-clustering strategies have been applied to interactome networks in order to identify functional modules and protein complexes as densely connected subgraphs $[18,19,20]$. In addition, new algorithms have specifically been designed for this task with the aim to distinguish real complex components from spurious interactors and to allow shared components across different complexes [1ㅡ, $\underline{22}]$. Various clustering techniques were also used to define protein complexes from purification data in the original largescale screens of macromolecular assemblies in yeast, fly, human and M. pneumoniae $[\underline{5}, \underline{6}, \underline{7}, \underline{8}, \underline{10}, \underline{11}]$. On the other hand, since protein complexes are often conserved due to the constraints PPIs pose on functional divergence during evolution [23,24], it is possible to predict complexes using orthology information. In its simplest form, orthology-based complex prediction reports the collection of all orthologous proteins of a given complex in one species as the corresponding complex in the other organism [25]. However, oneto-many and many-to-many orthology relationships between species often imply functional divergence of paralogous genes after duplication, leading to the prediction of many false complex components with increasing proteome size.

The recent advent of tools for the comparison and alignment of protein interaction networks $[\underline{26}, \underline{27}, \underline{28}, \underline{29}, \underline{30}, \underline{31}, \underline{32}]$ now opens up new possibilities for complex prediction. One strategy is to align whole interactome networks of different species to search for conserved functional modules $[\underline{27}, \underline{32}, \underline{33}, \underline{34}]$. For instance, Sharan et al. aligned the yeast and $H$. pylori interactomes, finding 11 conserved protein complexes [27], while Hirsh and colleagues found 150 conserved complexes by aligning the yeast and fly interactomes, matching known complexes in yeast with coherent functional annotations [33]. However, interactome to interactome alignment does not exploit knowledge about the composition of known complexes. This can only be done through complex to interactome alignment, in which the network representation of a known query complex in a given organism such as yeast is aligned to the interactome of a target species. For instance, Dost et al. developed the QNet algorithm [35], which allows the querying of input graphs of treelike topology in interaction networks, and used it to align 94 manually 
curated yeast complexes from the MPACT database [1] to the fly interactome, finding 36 of them to be conserved in fly.

For this work, we applied our recently developed tool for network comparison, NetAligner, which was demonstrated to outperform the current standard in the field in a variety of different benchmarks [36] and was already used successfully to discover the role of structural disorder in the rewiring of interactomes during evolution [국. Using NetAligner, we investigated how the incorporation of interaction data through network alignment influences the performance of orthology-based complex prediction. Moreover, we systematically aligned known protein complexes in $H$. sapiens, $S$. cerevisiae and $M$. pneumoniae to whole species interactomes to find novel complex components in yeast and human, predict yet undiscovered complexes in the fly $D$. melanogaster and search for similarities and differences between the complexes repertoires of $H$. sapiens and the human pathogen M. pneumoniae.

\section{Results and Discussion}

\section{Network alignment increases precision in orthology-based complex prediction}

A standard, straightforward method to predict protein complexes in a target species based on those known in a given query organism is the so-called 'orthologs approach'. In that method, the union of all orthologous proteins of the respective query complex components is predicted to constitute that complex in the target species [25]. The general idea behind this approach is that many protein complexes are evolutionarily conserved, because they perform critical cellular tasks, such as replication, transcription or translation, needed in all forms of cellular life. However, due to evolutionary divergence of proteins after duplication, which can lead to functionally non-overlapping paralogs, not all orthologs of the components of a given complex in one species should be expected to be part of the corresponding complex in another organism. Prediction of protein complexes using the standard orthologs approach can thus result in false complex components (i.e. false positive predictions). To test whether the incorporation of interaction information through network alignment can decrease the number of false positives and thus increase the precision of orthology-based complex prediction, we 
compared the performance of the orthologs approach to that of NetAligner [ $\underline{36}$ ] in predicting yeast protein complexes based on human complexes and vice versa through complex to interactome alignment (Fig. 1A). For this, we used the non-redundant benchmark set of 71 matching human-yeast complex pairs (see Materials \& Methods), which we previously defined [36], and analysed the complex predictions of the orthologs approach with respect to how well they agree with the corresponding benchmark set complexes (see Materials \& Methods). We evaluated both precision (i.e. fraction of true complex components among all proteins predicted to be part of the given complex) and recall (i.e. fraction of complex components recovered in the given prediction) in the same way as in our previous study [ㅎ6], so that we could directly compare the performance of the orthologs approach to the performance reported for NetAligner in predicting protein complexes via complex to interactome alignment (see below and Materials \& Methods). We found that incorporating interaction data through network alignment significantly increases the precision of orthology-based complex prediction (i.e. the ability to distinguish orthologs that are part of the complex in the target species from those that are not). When using NetAligner with default parameters there is a significant increase in precision from $34.6 \%$ to $54.1 \%$ ( $p$-value $=2.04 \times 10^{-32}$, one-sided Fisher's exact test), which is also present when calibrating NetAligner for the alignment of human complexes to the yeast interactome $\left(53.9 \%, p\right.$-value $=4.07 \times 10^{-32}$, one-sided Fisher's exact test) or vice versa $\left(58.5 \%, p\right.$-value $=2.65 \times 10^{-36}$, one-sided Fisher's exact test; Fig. 1B). This increase in precision arises from NetAligner using protein-protein interaction data to identify and filter out false positive orthology mappings. Due to current interactome networks still being incomplete and missing many interactions existing in nature [38], this filtering step unavoidably leads to an increase in false negatives and thus a reduced recall of true complex components (from $58.0 \%$ to $44.9 \%$ with default parameters; Fig. 1B). This is caused by orthologous proteins getting filtered out of complex predictions, since they seem not to interact with any other complex component, while those interactions indeed do exist and are just missing from current interactome networks. So currently, there is a tradeoff between precision and recall when using NetAligner to predict protein complexes. However, once species interactomes become more complete, we would expect network alignment approaches to deliver increased precision at the same level of recall of simple orthology mapping. Ongoing and future 
140 interaction detection experiments should increase interactome coverage and thus also 141 increase the recall of true complex components when predicting protein complexes 142 through network alignment.

143

144

\section{Strategy to predict complexes through complex to interactome alignment}

To predict complexes in yeast, human and fly, which are the species with the most interaction data available, and to identify novel complex components, we aligned the non-redundant sets of human, yeast and mycoplasma protein complexes (see Materials \& Methods) to whole species interactomes using NetAligner [36]. In contrast to a pure orthologs-approach, network alignment via NetAligner incorporates knowledge about protein-protein interactions into orthology-based complex prediction. When aligning a query complex to the interactome of a target species, NetAligner aligns those pairs of orthologous proteins that are part of the two input networks and identifies conserved and likely conserved interactions, as well as parts where the query complex and target interactome differ slightly, represented through gaps and mismatches in the alignment graph constructed by the program [36]. Several program parameters, such as the vertex and edge probability thresholds, further determine which pairs of orthologous proteins will be part of the final alignment solutions. For those alignment scenarios that were part of the complex to interactome alignment benchmark reported in [36] (i.e. yeast to human and human to yeast), we could use the best-performing parameter combinations, while for the others there is no benchmark data available, and we thus used the default parameters (Table 1). We considered only the highest-ranked significant alignment solution (with a standard p-value threshold of 0.05 ) for each query complex.

\section{Finding novel components of yeast and human protein complexes}

To find novel components of protein complexes in yeast and human, we aligned the nonredundant sets of 1027 human complexes and 244 yeast complexes from the manually curated databases CORUM [2] and MPACT [1], to the yeast and human interactome, respectively (see Materials \& Methods). Here, we did not use the complexes identified in large-scale studies to ensure that our predictions are based only on curated data sources. This yielded 257 non-redundant significant complex predictions in yeast and 89 in human (Table 2 and Fig. $2 \mathrm{~A} \& 2 \mathrm{C}$ ). We then identified novel components by comparing our complex predictions to all known complexes in the respective species 
(see Materials \& Methods). We found 307 non-redundant novel components across 181 yeast complexes and 175 non-redundant novel components in 65 human complexes (Table 2 and Fig. 2B \& 2D). Given the recall of the method when aligning human complexes to the yeast interactome and vice versa (Table 1), most of the predicted complexes are probably sub-complexes (as our method misses some complex components due to incomplete interaction data). Moreover, based on the precision of our method (Table 1), we can estimate that at least $60 \%$ of the novel yeast complex components we predicted (from aligning human complexes to the yeast interactome) and $49 \%$ of the novel human components we predicted (from aligning known yeast complexes to the human interactome) are real complex members. For an independent in silico validation of our predictions, we computed the number of predicted complexes that are functionally homogeneous (see Materials \& Methods) separately for each Gene Ontology (GO) category (i.e. biological process, molecular function and cellular component) [39], and compared it to the respective number of functionally homogeneous query complexes and predictions based on simple orthology mapping (Fig. 3A \& 3B). We found that, even when requiring the complexes to fulfill this criterion in at least two GO categories (e.g. 'biological process' and 'molecular function'; see Materials \& Methods), the majority of the predicted complexes in yeast $(70 \%)$ and human $(73 \%)$ are indeed functionally homogeneous. The fact that the fraction of human query complexes that are homogeneous is smaller than the fraction of predicted yeast complexes (63\% vs. $70 \%)$ might indicate that our predictions are, on average, of similar quality as the manually-curated human complexes stored in the CORUM database [2]. When using a simple orthologs approach instead of NetAligner, we got a similar fraction of homogeneous complex predictions in yeast $(73 \%)$. On the other hand, the fraction of homogeneous yeast query complexes is larger than the fraction of homogeneous complex predictions in human $(97 \%$ vs. $73 \%)$, suggesting that in this case, our predictions are of lower quality than the query complexes. However, when using a simple orthologs approach, the resulting fraction of homogeneous complex predictions in human is even lower with only 58\% (Fig. 3A \& 3B). A likely explanation for these results is that our current knowledge about protein complexes is considerably better in yeast than in human, with several real human complex components still missing the respective functional annotations and thus leading to less homogeneous complex predictions. Nevertheless, in most cases we tested, using NetAligner led to a higher 
204 fraction of homogeneous complex predictions than a simple orthologs approach (Fig. 3).

205 In addition, we tested whether the annotations of the predicted complexes hint towards

206 specific functional roles in the cell by computing the statistical significance of GO term 207 enrichments in each complex (see Materials \& Methods). We found that 233 of our 208 complex predictions in yeast $(91 \%)$ and 87 in human $(98 \%)$ were significantly 209 functionally enriched with respect to the given species interactome, indeed suggesting 210 specific cellular roles for those complexes and further validating our predictions. For the complete list of predicted complexes, see Supplemental Table 1, in which we annotated the complexes with the GO terms that are shared by most complex components to provide information about their possible biological roles and highlighted those functional annotations that are significantly enriched in the given complex. We also created subsets of high-confidence (HC) predictions (Supplemental Table 1), requiring each member complex to be functionally homogeneous and originate from a homogeneous query complex with which it shares at least one homogeneous GO term (see Materials \& Methods). This resulted in HC sets of 105 yeast and 61 human complex predictions (Table 2 and Fig. 2A \& 2C), with $98 \%$ and $97 \%$ of them, respectively, being significantly functionally enriched with respect to the given species interactome. We found 107 nonredundant novel components across $63 \mathrm{HC}$ yeast complexes and 91 non-redundant novel components in $41 \mathrm{HC}$ human complexes (Table 2 and Fig. 2B \& 2D). For instance, we predicted the proteins MPPA, MPPB, QCR1 and QCR2 to form a complex in human (Fig. 4A). All four proteins are orthologous to the alpha and beta subunits of the Mitochondrial processing peptidase (MPP) complex in yeast, which is involved in the maturation of mitochondrial proteins by proteolytic cleavage of the $\mathrm{N}$-terminal localization sequence [40]. NetAligner found the interaction between MPPA and MPPB of the yeast query complex to be conserved between human MPPA and MPPB, as well as between the QCR1 and QCR2 orthologs (also known as UCR-1 and UCR-2). In addition, this interaction was predicted to be likely conserved between the other components of the complex (Fig. 4A), but no subcomplex of any of the four proteins was found in current databases. According to our in silico validation experiments, all components of the predicted complex are involved in proteolysis and have metalloendopeptidase activity, but two of them (MPPA and MPPB) localise to the 235 mitochondrial matrix, while the other two (QCR1 and QCR2) localise to the 236 mitochondrial inner membrane as core components of the Cytochrome bc1 complex. 
Although it might be that the MPP and QCR subunits form two separate complexes in vivo, a combined MPP/QCR complex might also exist, since the two subcellular localizations are adjacent, and it was observed that in plants, the MPP complex is actually integrated into the cytochrome bc1 complex, with QCR1 and QCR2 being identical to MPPB and MPPA, respectively [40]. Another interesting example is the alignment of the human EXO1-MLH1-PCNA complex, which is involved in DNAmismatch repair, to the yeast interactome (Fig. 4B). The yeast complex predicted by the alignment solution consists of six different proteins, PCNA, RAD27, DIN7, EXO1, MLH1 and $\mathrm{MLH} 3$, based on interactions existing either in human or yeast and predicted to be likely conserved in the other species (Fig. 4B). The DNA sliding clamp PCNA and the endonuclease RAD27 (also known as FEN1) are known to form a complex in DNA replication and repair [41], and the interaction between MLH1 and MLH3 plays an important role in meiotic recombination and mismatch repair [42]. In addition, the double-stranded DNA exonucleases EXO1 and DIN7, both participating in mismatch repair, have high sequence similarity, and the double knockout of EXO1 and RAD27 is lethal [43]. Together, these findings point towards the possibility of a six-component mismatch-repair complex in yeast and also that the corresponding human complex might have additional components.

Finally, comparing the distributions of the total number of predicted (Fig. 2A \& 2C) and novel components (Fig. 2B \& 2D), we found that both achieve higher values in yeast than in human. However, this likely originates from the set of human query complexes simply being considerably larger (1027 complexes) than the set of yeast query complexes (244 complexes) and thus leading to both more complex predictions and a higher total number of novel components in yeast. On the other hand, the exponential decrease in the number of novel yeast complex components we observed (Fig. 2B) compared to the considerably broader distribution of novel human complex components (Fig. 2D), supports the view that our knowledge about yeast complexes surpasses that of human ones. This is because, relative to complex predictions in yeast, a larger fraction of predicted human complexes contains a given number of novel components. For instance, only 24 out of 257 predicted yeast complexes (9.3\%) contain three novel components, while the same is true for 16 out of 89 predicted human complexes (18\%). 


\section{Predicting fly complexes from human and yeast data is complementary rather}

\section{than redundant}

The fruitfly Drosophila melanogaster is an important model organism. However, there does not yet exist a dedicated database of protein complexes in fly, the first large-scale screen, reporting 556 complexes, has only recently been completed [ $\underline{9}$ ], and only 221 complexes are annotated in GO [39]. We thus predicted protein complexes in that species by aligning the non-redundant sets of 244 yeast complexes and 1027 human complexes to the fly interactome (see Materials \& Methods). This resulted in 66 nonredundant significant complex predictions originating from yeast and 219 from human (Table 3 and Fig. 2E \& 2G), with only little overlap (see below). Compared to the set of 777 known complexes in fly (based on GO annotations and the recent large-scale screen by Guruharsha et al. [9]; see Materials \& Methods), our complex predictions contained 252 non-redundant novel components across 56 complexes based on yeast data and 454 non-redundant novel components in 200 complexes originating from human data (Table 3 and Fig. $2 \mathrm{~F} \& 2 \mathrm{H}$ ). Among those complexes, 6 and 45, respectively, were entirely novel. Again based on the recall and precision of the method (Table 1), we can estimate that most of the predicted complexes are probably subcomplexes and that at least $54 \%$ of the novel components we found are real complex members. The independent in silico validation (see Materials \& Methods) revealed that the majority of the predicted fly complexes are functionally homogeneous, independent of whether they originated from yeast $(70 \%)$ or human (68\%) query complexes (Fig. 3C \& 3D). This indicates that both of those organisms represent interesting sources for predicting evolutionary conserved protein complexes in fly based on network alignment. The fraction of homogeneous predicted fly complexes also lies between that of the human $(63 \%)$ and yeast $(97 \%)$ query complexes, indicating that our predictions are, on average, of at least the same quality as the manually-curated human complexes stored in the CORUM database [2], but of lower quality than the manually-curated yeast complexes in MPACT [1]. Here, we observed the biggest loss of homogeneity in the cellular component category (Fig. 3C). This might, however, result from the respective fly proteins missing sub-cellular annotations, which would again suggest that the current knowledge about protein complexes in yeast surpasses that of complexes in other species. Using a simple orthologs approach instead of NetAligner again leads to lower fractions of homogeneous complex predictions, independent of whether they originated 
from yeast $(58 \%)$ or human $(60 \%)$ query complexes. Among the fly complex predictions, 58 based on yeast $(88 \%)$ and 189 based on human data $(86 \%)$ were significantly functionally enriched with respect to the fly interactome (see Materials \& Methods), indicating specific cellular roles for those complexes and further validating our predictions. For the complete list of all predicted fly complexes, see Supplemental Table 2. The HC subsets of fly complex predictions (see Materials \& Methods and Supplemental Table 2) consist of 42 fly complexes originating from yeast and 71 from human data (Table 3 and Fig. 2E \& 2G), with $95 \%$ and $94 \%$ of them, respectively, being significantly functionally enriched with respect to the fly interactome. We found 134 nonredundant novel components across $32 \mathrm{HC}$ complexes predicted from yeast and 168 non-redundant novel components in $62 \mathrm{HC}$ complexes originating from human query complexes, with 4 and 11 of those complexes being completely novel (Table 3 and Fig. $2 \mathrm{~F} \& 2 \mathrm{H}$ ). For instance, aligning the yeast Replication factor C (RFC) complex, consisting of the components RFC1-5, to the fly interactome predicted the corresponding fly assembly to encompass six proteins, Q9VKW3, Q9VX15, RFC1, RFC2, Q7KLW6 and Q9U9Q1 (Supplemental Fig. 1). According to the complex annotations in GO [39], the first four of those are known to belong to the RFC complex in fly, while the latter two are novel. Our alignments showed that both Q7KLW6 and Q9U9Q1 are orthologous to RFC2-5 in yeast, and our in silico validation experiments revealed that all six predicted components are involved in DNA replication and have ATP-binding capability, important for performing the loading of the DNA sliding clamp $[44,45]$. Moreover, in the HTP screen of Guruharsha et al. [9], the first five of those proteins were purified together, which provides further evidence for Q7KLW6 to actually be a component of the RFC complex in fly. On the other hand, this is also an example where NetAligner (through the incorporation of interaction data) was able to filter out false positive components that a simple orthologs approach would have predicted to belong to the complex. These comprise the proteins Q8T3K3, Q8IQ05 and Q95WV5, which are annotated as DNA replication accessory factors and most similar to the yeast chromosome transmission fidelity protein 18 (CTF18), which is known to substitute RFC1 in the RFC-like complex that also contains the proteins CTF8 and DCC1 and is required for establishment of chromosome cohesion in the S-phase of the cell cycle $[46,47]$. Moreover, the SNX subcomplex of the human Retromer complex, which is involved in mediating endosome to trans-Golgi network retrograde transport $[\underline{48}, \underline{49}]$, 
represents an example for predicting fly complexes based on human data (Fig. 4C). It consists of the SNX1-SNX6 dimer that is important for membrane-bound coat formation [48,49], and we predict this complex to exist in fly as an assembly of Q9VQQ6 and Q9VLQ9. This prediction is not only supported by SNX1 and SNX6 being the best BLAST [50] hits of Q9VQQ6 and Q9VLQ9, respectively, but also through the interaction between the two human components being conserved in fly (Fig. 4C). Together, the examples shown in Fig. 4 illustrate the fact that query complexes need not be fully connected (i.e. can contain isolated proteins), but rather that NetAligner is indeed capable of identifying conserved protein complexes despite the incompleteness of current interactome networks [38].

Comparing the fly complex predictions from yeast and human data (Fig. 5), we found only 26 pairs of matching complexes (i.e. pairs of predicted fly complexes with a component overlap of more than $50 \%$ of each complex), covering 21 non-redundant predicted complexes from human (10\%) and 12 from yeast data (18\%). One reason for this little overlap between the fly complex predictions is probably the low number of matching human and yeast query complexes. Indeed, only 41 (4\%) and $46(19 \%)$ of the non-redundant sets of 1027 human and 244 yeast query complexes, respectively, are present in the non-redundant set of matching human/yeast complex pairs. Between the $\mathrm{HC}$ subsets, there are only 11 pairs of matching complexes, covering 11 non-redundant predicted HC complexes from human (15\%) and 7 from yeast data (17\%; Fig. 5). Moreover, none of the completely novel complexes we predicted was found based on both yeast and human data. This clearly indicates that predicting fly complexes from yeast and human query complexes through network alignment is complementary rather than redundant. Protein complexes that were found both when starting from human and from yeast data include the Replication factor C complex, the Casein kinase II, the 20S core and 19/22S regulatory particles of the proteasome, as well as the Septin, Tubulin and Actin filament complexes (Fig. 5), which all represent well-studied conserved assemblies.

\section{Mycoplasma complexes differ substantially from those of eukaryotes}

Kühner et al. [] ] reported the first genome-wide analysis of protein complexes in the human pathogen Mycoplasma pneumoniae, which has one of the smallest known genomes (689 protein-encoding genes). This analysis revealed a rather complicated 
machinery of almost 200 protein complexes, of which the majority were yet unknown [8]. To predict whether some of these are actually conserved in other organisms, we aligned the non-redundant set of 174 mycoplasma complexes to the interactomes of yeast, fly and human (see Materials \& Methods). The complex to interactome alignments led to only 11,9 and 6 non-redundant significant predictions in those species, respectively (Table 4). Compared to the sets of known protein complexes (see Materials \& Methods), our predictions contained 86 non-redundant novel components across 6 yeast complexes, 68 across 9 fly complexes and 30 in 5 human complexes (Table 4). Based on the recall and precision of the method (Table 1), we can again estimate that most of the predicted complexes are probably sub-complexes and that at least $54 \%$ of the novel components we found are real complex members. According to our independent in silico validation (see Materials \& Methods), only $55 \%, 44 \%$ and $33 \%$ of all predicted yeast, fly and human complexes, respectively, are functionally homogeneous. If the mycoplasma interactome (on which the query complexes are based) contained many false positive interactions, one potential reason for the observed low functional homogeneity could be that these complex predictions are less reliable. Other possible reasons include inherent differences in the complexes repertoires of those species or aspects of biology that are less well studied. In contrast, the fact that only $39 \%$ of all query mycoplasma complexes are homogeneous is likely due to a lack of functional annotations in that organism.

Overall, since we used only the high-confidence subset of mycoplasma interactions (to reduce the number of false positives), we think the very low numbers of significant complex predictions (independent of the target species) and their low functional homogeneity suggest that the mycoplasma interactome (at least the part currently known) is very different from the interactomes of yeast, fly and human. This indicates that there probably exist protein complexes that are unique to the pathogen and might thus be targeted by drugs without causing adverse effects in the human host.

All complex predictions, except for one in yeast, were significantly functionally enriched with respect to the given species interactome, suggesting that they perform specific biological roles. For the complete list of predicted yeast, fly and human complexes, based on mycoplasma data, see Supplemental Table 3. The HC subsets of those complex predictions (see Materials \& Methods and Supplemental Table 3), consist of only three predicted complexes in yeast (one novel component), one in fly (four novel components) and one in human (no novel components; Table 4), with all of them being 
significantly functionally enriched with respect to the given species interactome. The mycoplasma query complexes that were predicted to be conserved in those $\mathrm{HC}$ sets comprise the DNA polymerase III complex in human, the ATP synthase complex in fly and yeast, as well as the 6-phosphofructokinase and the ribonucleoside-diphosphate reductase in yeast. The DNA polymerase III complex, consisting of DPO3X and the yet uncharacterized proteins Y007 and Y450, represents an interesting case: it was aligned to the RFC complex in human, whose clamp loading function is actually incorporated into the DNA polymerase III holoenzyme complex in prokaryotic species such as mycoplasma [1] .

Comparing the different subsets of mycoplasma complexes that led to significant predictions in the other species (Fig. 6), one complex, the Restriction enzyme complex, could be aligned to protein complexes in all three species interactomes, two additional complexes were found in both yeast and human (the DNA polymerase III and Cohesinlike complexes), two in both fly and human (the Protein chaperone and Pyruvate dehydrogenase complexes), and four complexes were found in both the yeast and fly interactome (the ATP synthase, Peptidase, Ribosome and RNA polymerase complexes; Fig. 6). This means that the majority of mycoplasma complexes that led to significant predictions $(56 \%)$ could be found in more than one target species, representing complexes involved in core biological processes conserved from bacteria up to higher eukaryotes. The alignment of the Restriction enzyme complex was, at first, surprising, because it is known to exist only in bacteria and archaea. A closer inspection, however, revealed that it was aligned to ATP-dependent metalloproteases in the eukaryotic species, suggesting that targeting the Restriction enzyme complex with drugs might also affect ATP-dependent metalloproteases in the human host and thus lead to potential adverse effects.

\section{Conclusions}

Protein complexes represent key molecular entities that are implicated in many important biological processes within a cell. However, complexes are yet largely uncharacterized in most species and experimental determination of their composition is still a costly endeavour. Increasing our knowledge about protein complexes in important 
model organisms via complex prediction thus represents an attractive option. Here, we showed that incorporating PPI data through network alignment significantly increases the precision of orthology-based complex prediction, though at the expense of missing some real complex components. By aligning known protein complexes to the interactomes of human, yeast and fly (the species with the highest number of interactions currently available in public databases) using NetAligner [36], our recently developed tool for network alignment, we were able to identify conserved protein (sub)complexes between human and yeast, as well as novel complex components, with higher precision than by using a simple orthologs-based approach. In addition, we predicted novel macromolecular assemblies (not present in current databases) in fly based on known yeast and human complexes, and found that our contemporary knowledge of yeast complexes surpasses that of other species, which is not surprising given the huge efforts invested into detecting macromolecular assemblies in yeast $[\underline{3}, \underline{4}, \underline{5}, \underline{6}, \underline{7}]$. On the other hand, we found that current human and yeast complex data leads to complementary predictions in fly, meaning that querying known macromolecular assemblies of those species in the fly interactome unveils different conserved complexes. In the in silico validation experiments that we performed, our NetAlignerbased complex predictions had about the same functional homogeneity as the known complexes we used for querying, and we were able to assign specific functional roles to almost all complexes. In contrast, predictions based on simple orthology mapping often resulted in reduced functional homogeneity. Finally, aligning the protein complexes of the human pathogen M. pneumoniae [] to the interactomes of human, yeast and fly yielded only a handful of significant complex predictions, suggesting that the mycoplasma interactome is at least in parts quite different from those of eukaryotic species. Quantifying those differences is very difficult due to the incompleteness of current interactome data [38]. Nevertheless, since this issue is at least partly addressed through the prediction of likely conserved interactions by the NetAligner algorithm, it indicates that some of the protein complexes that have been identified in mycoplasma might not have any counterpart in eukaryotes and thus represent interesting drug targets with little chances of causing adverse effects in the human host. On the other hand, our analyses revealed that the pathogen's Restriction enzyme complex, which is known to be unique to bacteria and archaea, is similar to ATP-dependent metalloproteases in human, implying that targeting this complex might have undesirable outcomes. 


\section{Materials \& Methods}

\section{Datasets of protein sequences}

We collected datasets of protein sequences for human (Homo sapiens), fly (Drosophila melanogaster), yeast (Saccharomyces cerevisiae) and mycoplasma (Mycoplasma pneumoniae) from the UniProt Knowledgebase release 15.8 [52] by merging the set of sequences stored in Swiss-Prot (including splice variants) and TrEMBL with experimental evidence on protein or transcript level. For mycoplasma, due to a lack of annotation data, we also included sequences not yet marked as having experimental evidence, but excluded all sequences that were only present in TrEMBL and thus of low confidence. Clustering based on $100 \%$ sequence identity resulted in non-redundant sets of 75,981 human, 23,296 fly, 6,121 yeast and 687 mycoplasma protein sequences.

\section{Lists of orthologous proteins}

We determined lists of orthologous proteins for species combinations of human, fly, yeast and mycoplasma by performing a reciprocal BLASTP [50] search. We used an Evalue threshold of $10^{-10}$ and considered only hits in the top10 of the BLASTP output to filter out spurious matches. This resulted in non-redundant sets of 91,112 human/fly, 19,558 human/yeast, 12,778 fly/yeast, 1,005 human/mycoplasma, 644 fly/mycoplasma and 488 yeast/mycoplasma orthologs.

\section{Interactome construction}

We built interactome networks for human, fly and yeast by extracting and merging binary protein-protein interaction data from the major interaction databases IntAct [53], MINT [54] and HPRD (for human) [55]. To increase the quality of the resulting binary interactome networks, we then filtered out all those interactions without support in form of Pubmed ID(s) or interaction detection method(s). For mycoplasma, we extracted the list of high-confidence, binary protein-protein interactions from Kühner et al. []], mapping ordered locus names to UniProt accession codes [52]. This resulted in non-redundant 
interactomes consisting of 53,290 interactions in human, 19,260 in fly, 60,721 in yeast and 1,058 in mycoplasma (Supplemental Table 4).

\section{Non-redundant benchmark set of human/yeast complex pairs}

We used the non-redundant benchmark set of conserved human/yeast complex pairs described in [36]. In brief, this set is based on the manually-curated yeast complexes from the MPACT database [1] and human complexes from the CORUM database [2] whose components are fully present in the respective species interactome, and clustered to remove redundancy. Matching (i.e. conserved) complexes between the two species are defined by requiring at least 2 and $25 \%$ of the components of the given human complex to have at least one ortholog in the respective yeast complex and vice versa. The complete benchmark set encompasses 71 matching human/yeast complex pairs, consisting of 64 non-redundant human and 52 non-redundant yeast complexes.

\section{Complex to interactome alignment using NetAligner}

We performed all complex to interactome alignments using the NetAligner algorithm that we recently developed [36] and which is also available as a web server [56]. For all species combinations, we computed vertex and interaction conservation probabilities required by NetAligner based on the interactomes and lists of orthologous proteins as described in [36], using default parameters. We assigned reliabilities to each interaction based on the number of Pubmed IDs supporting it as previously described [26]. For aligning the complexes to whole organism interactomes, we created a network representation of each complex, consisting of all interactions between complex components that are present in the respective species interactome and including selfinteractions with a reliability of 0 for all singletons in order to not lose any information about complex composition [흐] .

\section{Performance comparison to simple orthologs approach}

To compare the performance of NetAligner to that of a simple orthologs approach, in which the set of all orthologs of the components of a given query complex are predicted to be part of the complex in the target species, we evaluated the results of this approach with respect to the benchmark set of protein complexes as described in [36]. In brief, the complex predictions are evaluated in terms of how well they agree with the respective 
matching benchmark set complexes based on the overlap of their protein components. For each complex predicted by the orthologs approach, we first determined the bestmatching benchmark complex of the same species by minimising the total number of unmatched components. A complex prediction was deemed to 'cover' a benchmark complex if it contained at least 2 and at least $50 \%$ of its components. We then calculated the number of true positives (TP) as the total number of distinct proteins common to any given complex prediction and the benchmark complex it covers; the number of false positives (FP) as the total number of distinct proteins that are part of any given complex prediction, but not present in the benchmark complex it covers; and the number of false negatives (FN) as the total number of distinct proteins present in any given benchmark complex, but not part of any complex prediction covering that complex. Next, we computed the performance of the orthologs approach in terms of precision and recall:

$$
\text { precision }=\frac{\mathrm{TP}}{\mathrm{TP}+\mathrm{FP}} ; \text { recall }=\frac{\mathrm{TP}}{\mathrm{TP}+\mathrm{FN}}
$$

Finally, we report the average precision and recall of predicting yeast complexes based on human protein complex data and vice versa to avoid parameter overfitting (Fig. 1B). For NetAligner, since we used the same performance evaluation strategy, as well as the same list of orthologs and set of benchmark complexes, we could directly take the performance results reported in our previous work for complex to interactome alignment [36]. We also evaluated the performance when using default parameters (Fig. 1B).

\section{Non-redundant sets of protein complexes in human, yeast and mycoplasma}

We collected non-redundant sets of protein complexes in human, yeast and mycoplasma. For this, we first extracted all human complexes from the CORUM database [2], the set of manually curated yeast complexes from the MPACT database [1], as well as all homo- and heteromeric mycoplasma complexes from Kühner et al. []]. Analogously to the procedure for constructing the non-redundant benchmark set of human/yeast complex pairs [ㅎ6], we then filtered out those complexes that were not fully present in the respective species interactome and clustered them based on the overlap of their components using complete linkage hierarchical clustering to remove redundancy. The distance $d\left(c_{1}, c_{2}\right)$ between two complexes $c_{1}$ and $c_{2}$ was defined as: 


$$
d\left(c_{1}, c_{2}\right)=1-\frac{\left|c_{1} \cap c_{2}\right|}{\max \left(\left|c_{1}\right|,\left|c_{2}\right|\right)},
$$

548 and we cut the resulting dendrogram using a distance threshold of 0.5 , such that each

549 pair of complexes in the same cluster share more than $50 \%$ of their components

550 (choosing the largest complex of each cluster as its representative). This resulted in 551 non-redundant sets of 1027 protein complexes in human, 244 in yeast and 174 in 552 mycoplasma.

553

\section{Identification of novel complex components}

To identify novel components in our complex predictions, we compared them with the set of known complexes of the respective species. We took all 1826 known human complexes from the CORUM database [2] , all 402 human complexes annotated in the Gene Ontology (GO) [39], as well as the 155 and 622 complexes from the recent highthroughput (HTP) studies of Hutchins et al. [10] and Havugimana et al. [11], respectively (3005 complexes in total). For yeast, we collected all 263 manually curated and all 871 HTP complexes from the MPACT database [1], which include the large-scale studies performed by Gavin et al. []ㅡ and Ho et al. [4]. We then added the 491 and 547 complexes defined in the HTP studies by Gavin et al. [్] and Krogan et al. [్], respectively, as well as all 350 yeast complexes annotated in GO and the recently published set of 720 yeast complexes from Babu et al. [7] (3242 complexes in total). Since there does not yet exist a dedicated database of protein complexes in fly, we determined the set of known fly complexes based on the set of HTP complexes reported by Guruharsha et al. [9] and shared GO annotations (child terms of 'macromolecular complex' (GO:0032991)), similar to [57], resulting in a total of 777 fly protein complexes. For each predicted complex in a given organism, we then determined the known complex of that species with the largest overlap in terms of protein components and marked all those proteins as novel that are not part of the known complex. 
biological process, molecular function and cellular component). Then, we calculated the GO homogeneity $h(c)$ of each complex $c$ per GO category, defined as the maximum fraction of protein components $p(c)$ that share the same GO term $t[\underline{58}]$ :

$$
h(c)=\max _{t} \frac{\left|p_{t}(c)\right|}{|p(c)|}
$$

We classified each complex with a GO homogeneity of higher than 0.5 as functionally homogeneous in the given GO category. Lastly, to increase the confidence level of all subsequent analyses, we defined all those complexes as functionally homogeneous that fulfilled this criterion in at least two GO categories.

\section{Statistical significance of functional enrichments}

For all most-abundant functional annotations of a given complex and GO category (i.e. those that contribute to its functional homogeneity in that category), we determined the statistical significance of their enrichments in the complex with respect to the given species interactome, using a one-sided Fisher's exact test with Bonferroni multiple testing correction and a strict $p$-value threshold of 0.025 . We then defined all those complexes as significantly functionally enriched that had a significant enrichment $p$ value in at least two GO categories.

\section{High-confidence subsets of complex predictions}

We post-processed all sets of predicted complexes to define high-confidence $(\mathrm{HC})$ subsets. A predicted complex has to fulfil the following criteria to be present in the given $\mathrm{HC}$ set: it has to (i) be part of the non-redundant subset of significant predictions, (ii) originate from a functionally homogeneous query complex, (iii) be homogeneous itself and (iv) share at least one homogeneous GO term with the given query complex. Query complexes were defined as high-confidence if they were functionally homogeneous.

\section{Acknowledgments}

We would like to thank Sebastian Kühner (Anne-Claude Gavin's lab at EMBL 
601

602

603

604

605

606

607

608

609

610

611

612

613

614

615

616

617

618

619

620

621

622

623

624

625

626

627

628

629

630

631

632

633

634

635

636

637

638

639

640

641

642

Amelie Stein (UCSF) and Andreas Zanzoni (University of Aix-Marseille) for helpful

discussions.

\section{References}

1. Güldener U, Münsterkötter M, Oesterheld M, Pagel P, Ruepp A, Mewes H-W, Stümpflen V (2006) MPact: the MIPS protein interaction resource on yeast. Nucleic Acids Res 34: D436-441.

2. Ruepp A, Waegele B, Lechner M, Brauner B, Dunger-Kaltenbach I, Fobo G, Frishman G, Montrone C, Mewes H-W (2010) CORUM: the comprehensive resource of mammalian protein complexes--2009. Nucleic Acids Res 38: D497-501.

3. Gavin A-C, Bösche M, Krause R, Grandi P, Marzioch M, Bauer A, Schultz J, Rick JM, Michon A-M, Cruciat C-M, Remor M, Höfert C, Schelder M, Brajenovic M, Ruffner H, Merino A, Klein K, Hudak M, Dickson D, Rudi T, Gnau V, Bauch A, Bastuck S, Huhse B, Leutwein C, Heurtier M-A, Copley RR, Edelmann A, Querfurth E, Rybin V, Drewes G, Raida M, Bouwmeester T, Bork P, Seraphin B, Kuster B, Neubauer G, Superti-Furga G (2002) Functional organization of the yeast proteome by systematic analysis of protein complexes. Nature 415: 141-147.

4. Ho Y, Gruhler A, Heilbut A, Bader GD, Moore L, Adams S-L, Millar A, Taylor P, Bennett K, Boutilier K, Yang L, Wolting C, Donaldson I, Schandorff S, Shewnarane J, Vo M, Taggart J, Goudreault M, Muskat B, Alfarano C, Dewar D, Lin Z, Michalickova K, Willems AR, Sassi H, Nielsen PA, Rasmussen KJ, Andersen JR, Johansen LE, Hansen LH, Jespersen H, Podtelejnikov A, Nielsen E, Crawford J, Poulsen V, Sørensen BD, Matthiesen J, Hendrickson RC, Gleeson F, Pawson T, Moran MF, Durocher D, Mann M, Hogue CWV, Figeys D, Tyers M (2002) Systematic identification of protein complexes in Saccharomyces cerevisiae by mass spectrometry. Nature 415: 180-183.

5. Gavin A-C, Aloy P, Grandi P, Krause R, Boesche M, Marzioch M, Rau C, Jensen LJ, Bastuck S, Dümpelfeld B, Edelmann A, Heurtier M-A, Hoffman V, Hoefert C, Klein K, Hudak M, Michon A-M, Schelder M, Schirle M, Remor M, Rudi T, Hooper S, Bauer A, Bouwmeester T, Casari G, Drewes G, Neubauer G, Rick JM, Kuster B, Bork P, Russell RB, Superti-Furga G (2006) Proteome survey reveals modularity of the yeast cell machinery. Nature 440: 631-636.

6. Krogan NJ, Cagney G, Yu H, Zhong G, Guo X, Ignatchenko A, Li J, Pu S, Datta N, Tikuisis AP, Punna T, Peregrín-Alvarez JM, Shales M, Zhang X, Davey M, Robinson MD, Paccanaro A, Bray JE, Sheung A, Beattie B, Richards DP, Canadien V, Lalev A, Mena F, Wong P, Starostine A, Canete MM, Vlasblom J, Wu S, Orsi C, Collins SR, Chandran S, Haw R, Rilstone JJ, Gandi K, Thompson NJ, Musso G, St Onge P, Ghanny S, Lam MHY, Butland G, Altaf-Ul AM, Kanaya S, Shilatifard A, O'Shea E, Weissman JS, Ingles CJ, Hughes TR, Parkinson J, Gerstein M, Wodak SJ, Emili A, Greenblatt JF (2006) Global landscape of protein complexes in the yeast Saccharomyces cerevisiae. Nature 440: 637643.

7. Babu M, Vlasblom J, Pu S, Guo X, Graham C, Bean BDM, Burston HE, Vizeacoumar FJ, Snider J, Phanse S, Fong V, Tam YYC, Davey M, Hnatshak O, Bajaj N, Chandran S, Punna T, Christopolous C, Wong V, Yu A, Zhong G, Li J, Stagljar I, Conibear E, Wodak 
SJ, Emili A, Greenblatt JF (2012) Interaction landscape of membrane-protein complexes in Saccharomyces cerevisiae. Nature 489: 585-589.

8. Kühner S, Van Noort V, Betts MJ, Leo-Macias A, Batisse C, Rode M, Yamada T, Maier T, Bader S, Beltran-Alvarez P, Castano-Diez D, Chen W-H, Devos D, Guell M, Norambuena T, Racke I, Rybin V, Schmidt A, Yus E, Aebersold R, Herrmann R, Böttcher B, Frangakis AS, Russell RB, Serrano L, Bork P, Gavin A-C (2009) Proteome Organization in a Genome-Reduced Bacterium. Science 326: 1235-1240.

9. Guruharsha KG, Rual J-F, Zhai B, Mintseris J, Vaidya P, Vaidya N, Beekman C, Wong C, Rhee DY, Cenaj O, McKillip E, Shah S, Stapleton M, Wan KH, Yu C, Parsa B, Carlson JW, Chen X, Kapadia B, VijayRaghavan K, Gygi SP, Celniker SE, Obar RA, ArtavanisTsakonas S (2011) A protein complex network of Drosophila melanogaster. Cell 147: 690703.

10. Hutchins JRA, Toyoda Y, Hegemann B, Poser I, Hériché J-K, Sykora MM, Augsburg M, Hudecz O, Buschhorn BA, Bulkescher J, Conrad C, Comartin D, Schleiffer A, Sarov M, Pozniakovsky A, Slabicki MM, Schloissnig S, Steinmacher I, Leuschner M, Ssykor A, Lawo S, Pelletier L, Stark H, Nasmyth K, Ellenberg J, Durbin R, Buchholz F, Mechtler K, Hyman AA, Peters J-M (2010) Systematic Analysis of Human Protein Complexes Identifies Chromosome Segregation Proteins. Science (New York, NY).

11. Havugimana PC, Hart GT, Nepusz T, Yang H, Turinsky AL, Li Z, Wang PI, Boutz DR, Fong V, Phanse S, Babu M, Craig SA, Hu P, Wan C, Vlasblom J, Dar V-U-N, Bezginov A, Clark GW, Wu GC, Wodak SJ, Tillier ERM, Paccanaro A, Marcotte EM, Emili A (2012) A census of human soluble protein complexes. Cell 150: 1068-1081.

12. Dezso Z, Oltvai ZN, Barabási A-L (2003) Bioinformatics analysis of experimentally determined protein complexes in the yeast Saccharomyces cerevisiae. Genome Research 13: 2450-2454.

13. Hart GT, Lee I, Marcotte ER (2007) A high-accuracy consensus map of yeast protein complexes reveals modular nature of gene essentiality. BMC bioinformatics 8: 236 .

14. Wang H, Kakaradov B, Collins SR, Karotki L, Fiedler D, Shales M, Shokat KM, Walther TC, Krogan NJ, Koller D (2009) A complex-based reconstruction of the Saccharomyces cerevisiae interactome. Molecular \& Cellular Proteomics 8: 1361-1381.

15. Pache RA, Babu MM, Aloy P (2009) Exploiting gene deletion fitness effects in yeast to understand the modular architecture of protein complexes under different growth conditions. BMC Systems Biology 3: 74.

16. Pache RA, Aloy P (2008) Incorporating high-throughput proteomics experiments into structural biology pipelines: identification of the low-hanging fruits. PROTEOMICS 8: 1959-1964.

17. Brooks MA, Gewartowski K, Mitsiki E, Létoquart J, Pache RA, Billier Y, Bertero M, Corréa M, Czarnocki-Cieciura M, Dadlez M, Henriot V, Lazar N, Delbos L, Lebert D, Piwowarski J, Rochaix P, Böttcher B, Serrano L, Séraphin B, van Tilbeurgh H, Aloy P, Perrakis A, Dziembowski A (2010) Systematic bioinformatics and experimental validation of yeast complexes reduces the rate of attrition during structural investigations. Structure (London, England : 1993) 18: 1075-1082.

18. Spirin V, Mirny LA (2003) Protein complexes and functional modules in molecular networks. Proceedings of the National Academy of Sciences of the United States of America 100: 12123-12128.

19. Pereira-Leal JB, Enright AJ, Ouzounis CA (2004) Detection of functional modules from protein interaction networks. Proteins 54: 49-57.

20. Poyatos JF, Hurst LD (2004) How biologically relevant are interaction-based modules in protein networks? Genome biology 5: R93. 
692

693

694

695

696

697

698

699

700

701

702

703

704

705

706

707

708

709

710

711

712

713

714

715

716

717

718

719

720

721

722

723

724

725

726

727

728

729

730

731

732

733

734

735

736

737

738

739

740

21. Bader GD, Hogue CWV (2003) An automated method for finding molecular complexes in large protein interaction networks. BMC bioinformatics $4: 2$.

22. Pu S, Vlasblom J, Emili A, Greenblatt J, Wodak SJ (2007) Identifying functional modules in the physical interactome of Saccharomyces cerevisiae. Proteomics 7: 944-960.

23. Roguev A, Bandyopadhyay S, Zofall M, Zhang K, Fischer T, Collins SR, Qu H, Shales M, Park H-O, Hayles J, Hoe K-L, Kim D-U, Ideker T, Grewal SI, Weissman JS, Krogan NJ (2008) Conservation and Rewiring of Functional Modules Revealed by an Epistasis Map in Fission Yeast. Science 322: 405-410.

24. van Dam TJP, Snel B (2008) Protein complex evolution does not involve extensive network rewiring. PLoS Computational Biology 4: e1000132.

25. Koonin EV, Wolf YI, Aravind L (2001) Prediction of the archaeal exosome and its connections with the proteasome and the translation and transcription machineries by a comparative-genomic approach. Genome Research 11: 240-252.

26. Kelley BP, Sharan R, Karp RM, Sittler T, Root DE, Stockwell BR, Ideker T (2003) Conserved pathways within bacteria and yeast as revealed by global protein network alignment. Proc Natl Acad Sci USA 100: 11394-11399.

27. Sharan R, Ideker T, Kelley B, Shamir R, Karp RM (2005) Identification of protein complexes by comparative analysis of yeast and bacterial protein interaction data. Journal of computational biology : a journal of computational molecular cell biology 12: 835-846.

28. Koyutürk M, Kim Y, Topkara U, Subramaniam S, Szpankowski W, Grama A (2006) Pairwise alignment of protein interaction networks. J Comput Biol 13: 182-199.

29. Sharan R, Ideker $T$ (2006) Modeling cellular machinery through biological network comparison. Nat Biotechnol 24: 427-433.

30. Cootes AP, Muggleton SH, Sternberg MJE (2007) The identification of similarities between biological networks: application to the metabolome and interactome. Journal of Molecular Biology 369: 1126-1139.

31. Kiemer L, Cesareni G (2007) Comparative interactomics: comparing apples and pears? Trends Biotechnol 25: 448-454.

32. Narayanan M, Karp RM (2007) Comparing protein interaction networks via a graph matchand-split algorithm. J Comput Biol 14: 892-907.

33. Hirsh E, Sharan R (2007) Identification of conserved protein complexes based on a model of protein network evolution. Bioinformatics 23: e170-176.

34. Ali W, Deane CM (2009) Functionally guided alignment of protein interaction networks for module detection. Bioinformatics 25: 3166-3173.

35. Dost B, Shlomi T, Gupta N, Ruppin E, Bafna V, Sharan R (2008) QNet: a tool for querying protein interaction networks. Journal of Computational Biology 15: 913-925.

36. Pache RA, Aloy P (2012) A novel framework for the comparative analysis of biological networks. PloS one 7: e31220.

37. Mosca R, Pache RA, Aloy P (2012) The role of structural disorder in the rewiring of protein interactions through evolution. Molecular \& Cellular Proteomics: 1-8.

38. Venkatesan K, Rual J-F, Vazquez A, Stelzl U, Lemmens I, Hirozane-Kishikawa T, Hao T, Zenkner M, Xin X, Goh K-I, Yildirim MA, Simonis N, Heinzmann K, Gebreab F, Sahalie JM, Cevik S, Simon C, de Smet A-S, Dann E, Smolyar A, Vinayagam A, Yu H, Szeto D, Borick H, Dricot A, Klitgord N, Murray RR, Lin C, Lalowski M, Timm J, Rau K, Boone C, Braun P, Cusick ME, Roth FP, Hill DE, Tavernier J, Wanker EE, Barabási A-L, Vidal M (2009) An empirical framework for binary interactome mapping. Nat Methods 6: 8390.

39. Ashburner M, Ball CA, Blake JA, Botstein D, Butler H, Cherry JM, Davis AP, Dolinski K, Dwight SS, Eppig JT, Harris MA, Hill DP, Issel-Tarver L, Kasarskis A, Lewis S, Matese 
JC, Richardson JE, Ringwald M, Rubin GM, Sherlock G (2000) Gene ontology: tool for the unification of biology. The Gene Ontology Consortium. Nature genetics 25: 25-29.

40. Nomura H, Athauda SBP, Wada H, Maruyama Y, Takahashi K, Inoue H (2006) Identification and reverse genetic analysis of mitochondrial processing peptidase and the core protein of the cytochrome bcl complex of Caenorhabditis elegans, a model parasitic nematode. Journal of biochemistry 139: 967-979.

41. Gomes XV, Burgers PM (2000) Two modes of FEN1 binding to PCNA regulated by DNA. The EMBO journal 19: 3811-3821.

42. Wang TF, Kleckner N, Hunter N (1999) Functional specificity of MutL homologs in yeast: evidence for three Mlh1-based heterocomplexes with distinct roles during meiosis in recombination and mismatch correction. Proceedings of the National Academy of Sciences of the United States of America 96: 13914-13919.

43. Tishkoff DX, Boerger AL, Bertrand P, Filosi N, Gaida GM, Kane MF, Kolodner RD (1997) Identification and characterization of Saccharomyces cerevisiae EXO1, a gene encoding an exonuclease that interacts with MSH2. Proceedings of the National Academy of Sciences of the United States of America 94: 7487-7492.

44. Gomes XV, Schmidt SL, Burgers PM (2001) ATP utilization by yeast replication factor C. II. Multiple stepwise ATP binding events are required to load proliferating cell nuclear antigen onto primed DNA. The Journal of biological chemistry 276: 34776-34783.

45. Schmidt SL, Gomes XV, Burgers PM (2001) ATP utilization by yeast replication factor C. III. The ATP-binding domains of Rfc2, Rfc3, and $\mathrm{Rfc} 4$ are essential for DNA recognition and clamp loading. The Journal of biological chemistry 276: 34784-34791.

46. Mayer ML, Gygi SP, Aebersold R, Hieter P (2001) Identification of RFC(Ctf18p, Ctf8p, Dcc1p): an alternative RFC complex required for sister chromatid cohesion in S. cerevisiae. Molecular cell 7: 959-970.

47. Naiki T, Kondo T, Nakada D, Matsumoto K, Sugimoto K (2001) Chl12 (Ctf18) forms a novel replication factor C-related complex and functions redundantly with Rad24 in the DNA replication checkpoint pathway. Molecular and cellular biology 21: 5838-5845.

48. Wassmer T, Attar N, Bujny MV, Oakley J, Traer CJ, Cullen PJ (2007) A loss-of-function screen reveals SNX5 and SNX6 as potential components of the mammalian retromer. Journal of cell science 120: 45-54.

49. Hong Z, Yang Y, Zhang C, Niu Y, Li K, Zhao X, Liu J-J (2009) The retromer component SNX6 interacts with dynactin p150(Glued) and mediates endosome-to-TGN transport. Cell research 19: 1334-1349.

50. Altschul SF, Madden TL, Schäffer AA, Zhang J, Zhang Z, Miller W, Lipman DJ (1997) Gapped BLAST and PSI-BLAST: a new generation of protein database search programs. Nucleic Acids Res 25: 3389-3402.

51. Pomerantz RT, O'Donnell M (2007) Replisome mechanics: insights into a twin DNA polymerase machine. Trends in microbiology 15: 156-164.

52. UniProt-Consortium (2009) The Universal Protein Resource (UniProt) 2009. Nucleic acids research 37: D169-174.

53. Aranda B, Achuthan P, Alam-Faruque Y, Armean I, Bridge A, Derow C, Feuermann M, Ghanbarian AT, Kerrien S, Khadake J, Kerssemakers J, Leroy C, Menden M, Michaut M, Montecchi-Palazzi L, Neuhauser SN, Orchard S, Perreau V, Roechert B, van Eijk K, Hermjakob H (2010) The IntAct molecular interaction database in 2010. Nucleic Acids Research 38: D525-531.

54. Ceol A, Chatr Aryamontri A, Licata L, Peluso D, Briganti L, Perfetto L, Castagnoli L, Cesareni G (2010) MINT, the molecular interaction database: 2009 update. Nucleic Acids Research 38: D532-539. 
790

791

792

793

794

795

796

797

798

799

800

801

55. Keshava Prasad TS, Goel R, Kandasamy K, Keerthikumar S, Kumar S, Mathivanan S, Telikicherla D, Raju R, Shafreen B, Venugopal A, Balakrishnan L, Marimuthu A, Banerjee S, Somanathan DS, Sebastian A, Rani S, Ray S, Harrys Kishore CJ, Kanth S, Ahmed M, Kashyap MK, Mohmood R, Ramachandra YL, Krishna V, Rahiman BA, Mohan S, Ranganathan P, Ramabadran S, Chaerkady R, Pandey A (2009) Human Protein Reference Database--2009 update. Nucleic Acids Res 37: D767-772.

56. Pache RA, Céol A, Aloy P (2012) NetAligner--a network alignment server to compare complexes, pathways and whole interactomes. Nucleic acids research.

57. Bruckner S, Hüffner F, Karp RM, Shamir R, Sharan R (2010) Topology-free querying of protein interaction networks. J Comput Biol 17: 237-252.

58. Goh K-I, Cusick ME, Valle D, Childs B, Vidal M, Barabási A-L (2007) The human disease network. Proc Natl Acad Sci USA 104: 8685-8690. 


\section{Figure 1}

Network alignment vs. simple orthologs approach in predicting protein complexes.

Comparison of network alignment (using NetAligner [36] ) and a simple orthologs approach in predicting protein complexes in a target species based on known complexes in a query organism. A: In the simple orthologs approach, all proteins in the target species (yellow) that are orthologous to the proteins of the query complex (violet) are predicted to form the given complex in the target organism. This can lead to false positives (proteins marked with red dashed lines) that are not part of the real complex. In the more sophisticated network alignment strategy, only those orthologs are predicted to be part of the given complex in the target species that are supported by conserved or likely-conserved interactions (red) between the two organism interactomes. B: Performance of the orthologs (grey) and NetAligner (blue) methods on a benchmark set of known conserved human/yeast complex pairs, using default parameters (default) or parameter sets trained on one species pair $(H / Y, Y / H)$ and evaluated across both species pairs. Error bars denote one standard error of the mean.

A

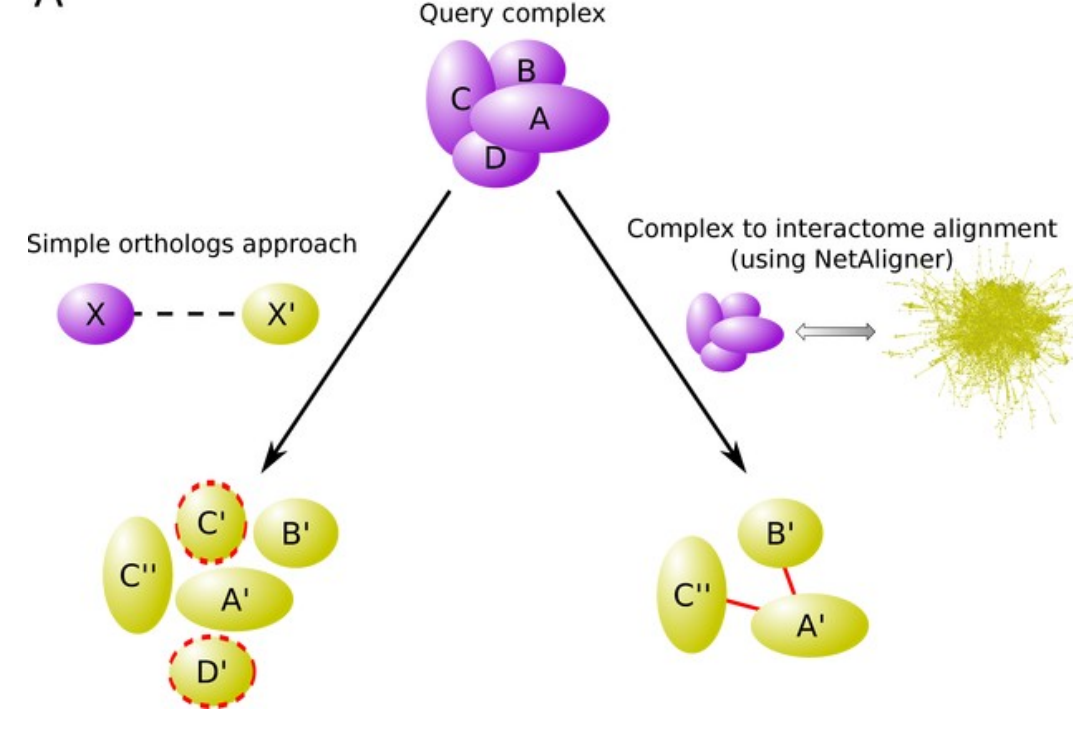

B

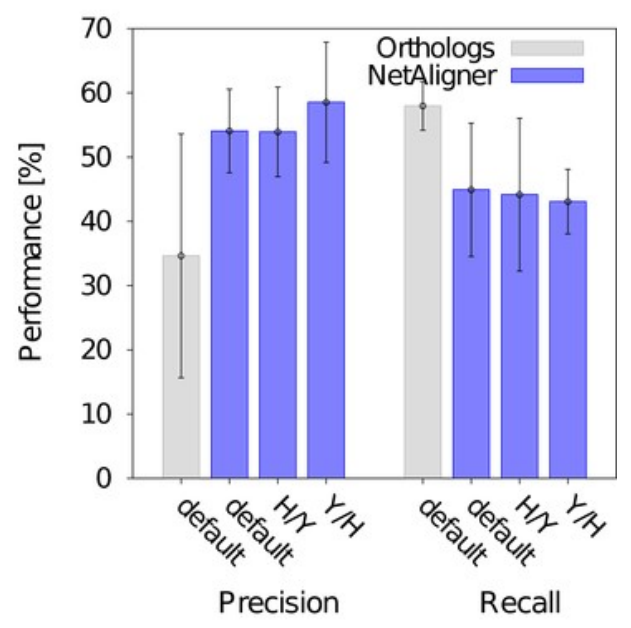




\section{Table $\mathbf{1}_{\text {(on next page) }}$}

NetAligner parameters and expected alignment performance.

The NetAligner parameters for complex to interactome alignment were taken from [36]. For yeast to human and human to yeast alignments, we took the best performing parameter combinations for these species as determined in the benchmarks [36]. For all other species, we used the default parameters for complex to interactome alignment. The option to predict likely conserved interactions is always set to true (1), because this considerably improves alignment performance [36]. Precision and recall describe the expected performance of NetAligner in correctly identifying protein complex components (see Materials \& Methods). 


\begin{tabular}{|c|c|c|c|c|}
\hline & \multicolumn{3}{|c|}{ Complex to interactome alignment } \\
\hline & & $\begin{array}{l}\text { Yeast to } \\
\text { human }\end{array}$ & $\begin{array}{l}\text { Human to } \\
\text { yeast }\end{array}$ & $\begin{array}{l}\text { Other } \\
\text { species }\end{array}$ \\
\hline \multirow{5}{*}{ 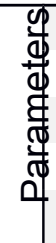 } & Predict likely conserved interactions & 1 & 1 & 1 \\
\hline & Vertex probability threshold & 0.0 & 0.0 & 0.0 \\
\hline & Edge probability threshold & 0.1 & 0.0 & 0.0 \\
\hline & Max insertion length & 1 & 2 & 2 \\
\hline & Vertex to edge score balance & 0.2 & 0.0 & 0.1 \\
\hline \multirow{2}{*}{ 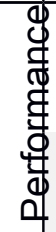 } & Precision [\%] & 49.17 & 60.91 & 54.07 \\
\hline & Recall [\%] & 38.06 & 56.06 & 44.91 \\
\hline
\end{tabular}




\section{Figure 2}

Complex size and number of novel components distributions for all predicted complexes.

Distributions of the total number of components of all predicted complexes (blue) and the high-confidence $(\mathrm{HC})$ subset (yellow) are shown on the left. Distributions of the number of novel components found in all predicted complexes (violet) and in the $\mathrm{HC}$ subset (orange) are shown on the right. A\&B: prediction of yeast complexes based on human data; C\&D: prediction of human complexes based on yeast data; E\&F: prediction of fly complexes based on yeast data; G\&H: prediction of fly complexes based on human data. 
Human to yeast

A

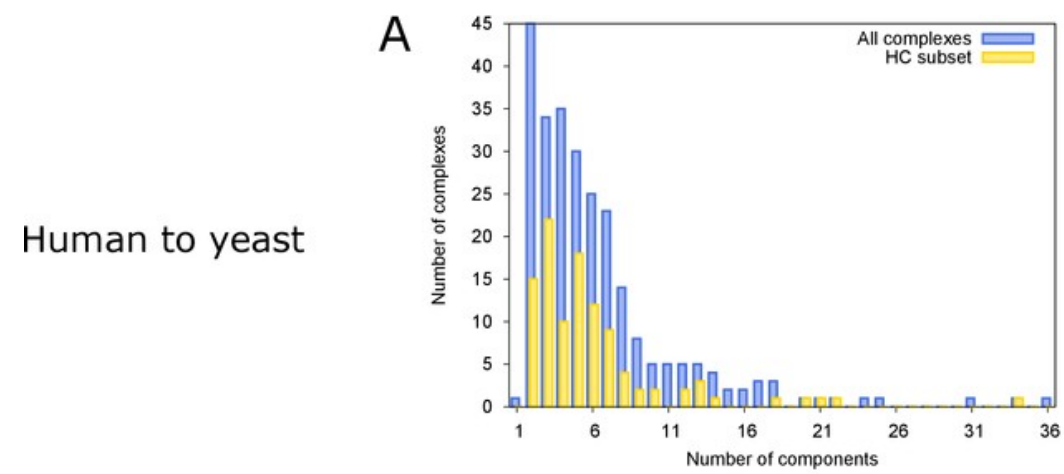

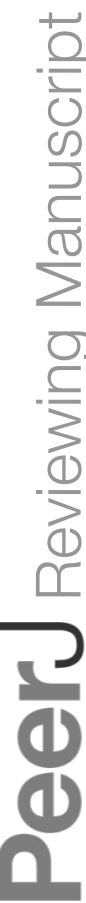

Yeast to human

C

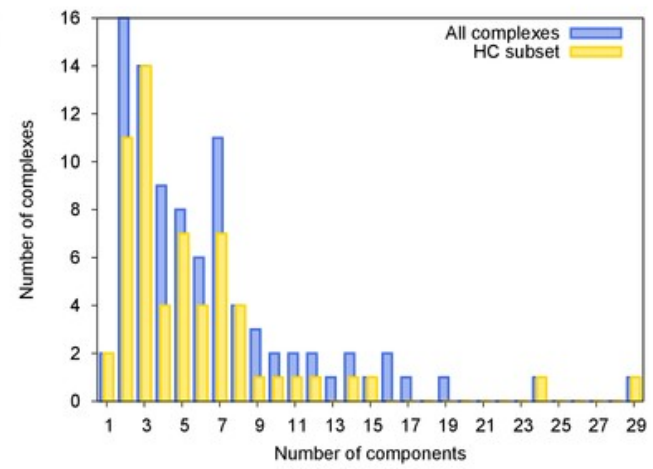

E

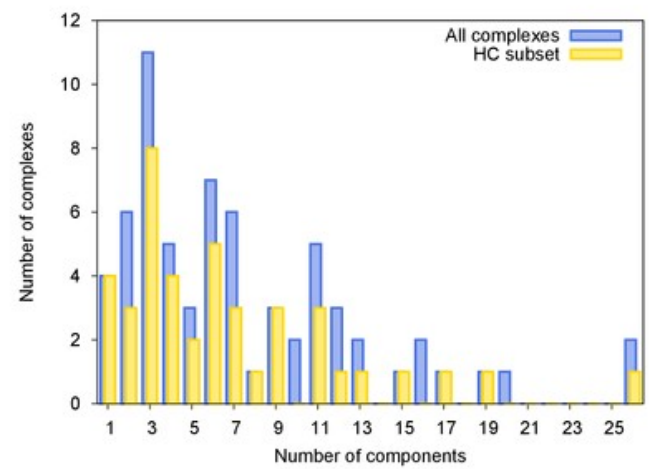

Human to fly

Yeast to fly

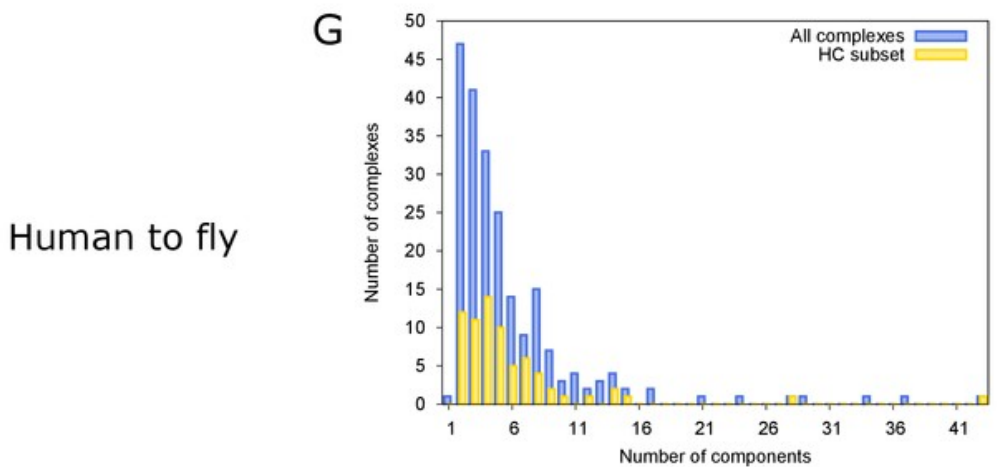

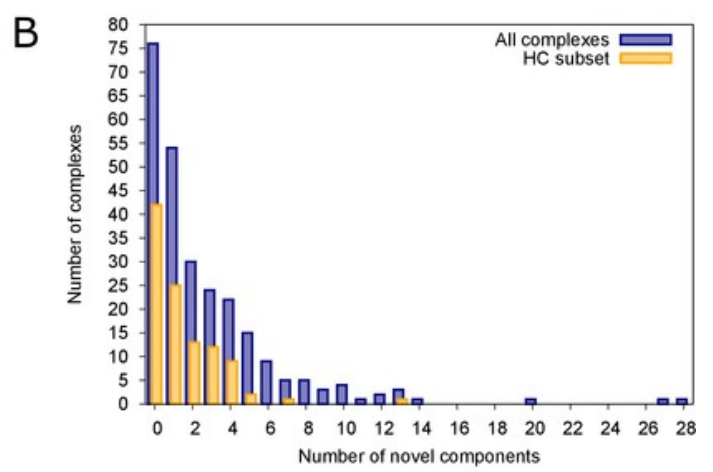

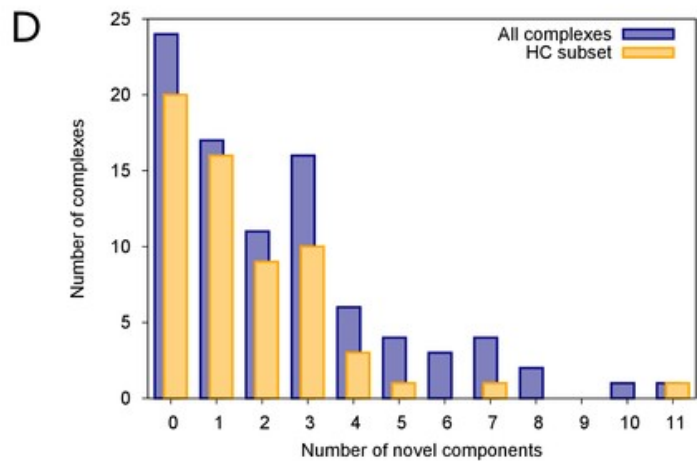

F

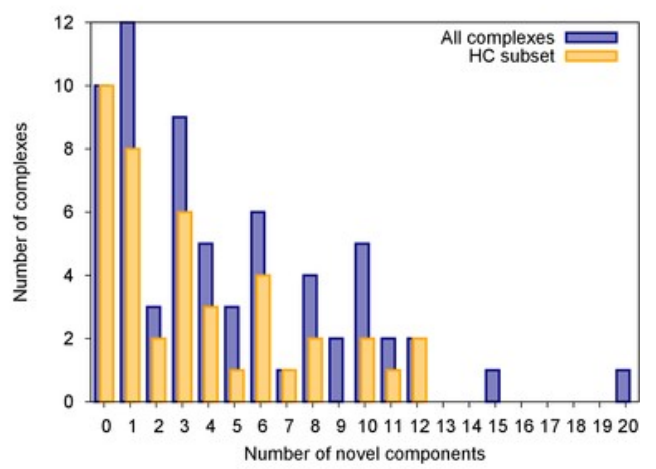

$\mathrm{H}$

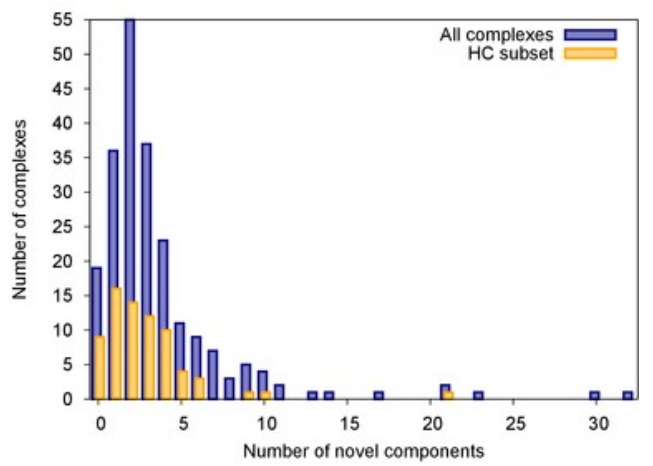




\section{Table 2 (on next page)}

Complex predictions in yeast and human.

Basic statistics of the complex prediction results in yeast and human, based on aligning known human complexes to the yeast interactome and vice versa (see Materials \& Methods). Results are shown both for all complex predictions (All) and for the high-confidence subset $(\mathrm{HC})$. \#, number of; nr, non-redundant. 


\begin{tabular}{l|c|c|c|c} 
& \multicolumn{2}{|c|}{ Human to yeast } & \multicolumn{2}{c}{ Yeast to human } \\
\hline Prediction type & All & HC & All & HC \\
\hline \# query complexes & 1027 & 645 & 244 & 236 \\
\hline \# predicted complexes (nr) & 257 & 105 & 89 & 61 \\
Total \# complex components (nr) & 604 & 372 & 464 & 325 \\
Average \# proteins per complex & 6.4 & 5.93 & 6.37 & 5.67 \\
Total \# novel components (nr) & 307 & 107 & 175 & 91 \\
Average \# novel components per complex & 2.75 & 1.46 & 2.4 & 1.62 \\
\# entirely novel complexes (nr) & 0 & 0 & 2 & 2
\end{tabular}




\section{Figure 3}

Functional homogeneity of query and predicted complexes.

Comparison of the functional homogeneity of query (grey) and predicted complexes, using either a simple orthologs approach (purple) or NetAligner (green), in the different Gene Ontology (GO) annotation categories. BP, Biological Process; CC, Cellular Component; MF, Molecular Function. A: prediction of yeast complexes based on human data; B: prediction of human complexes based on yeast data; C: prediction of fly complexes based on yeast data; D: prediction of fly complexes based on human data. 


\section{A Human to yeast}

Query complexes

Predicted complexes (Orthologs approach)

Predicted complexes (NetAligner)

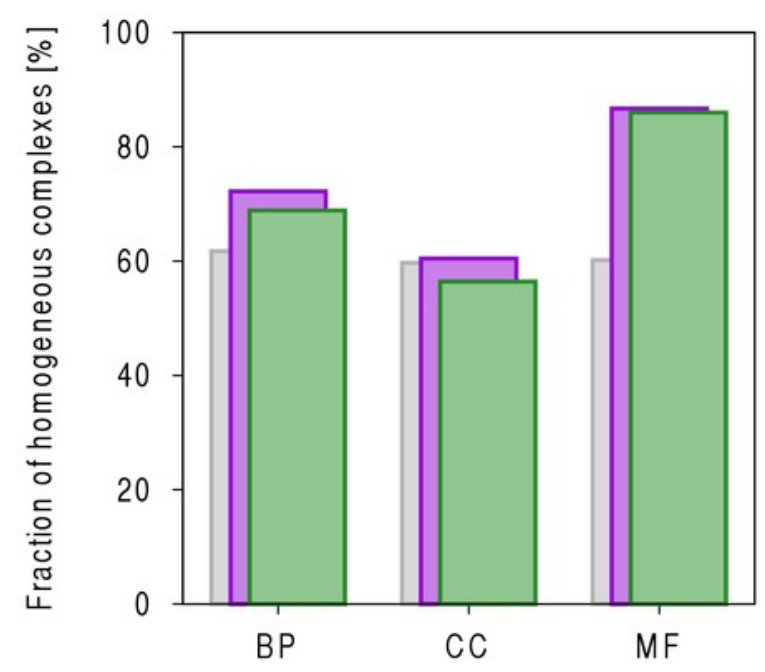

C

Yeast to fly

Query complexes

Predicted complexes (Orthologs approach)

Predicted complexes (NetAligner)

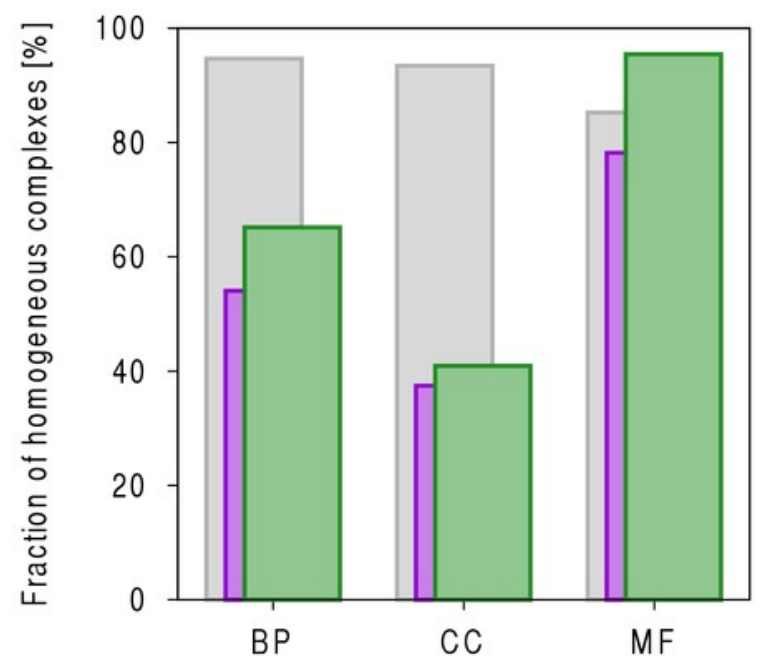

B Yeast to human

Query complexes

Predicted complexes (Orthologs approach)

Predicted complexes (NetAligner)

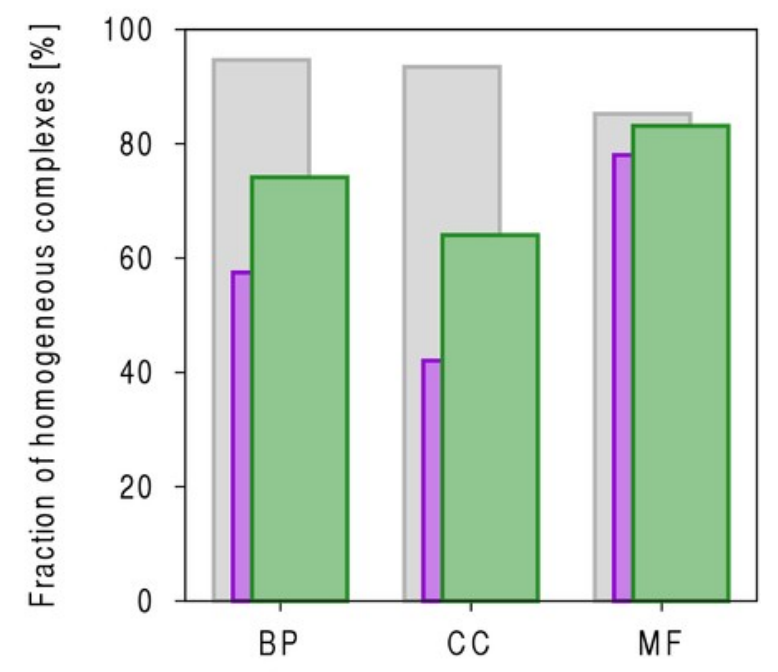

D Human to fly

Query complexes Predicted complexes (Orthologs approach)

Predicted complexes (NetAligner)

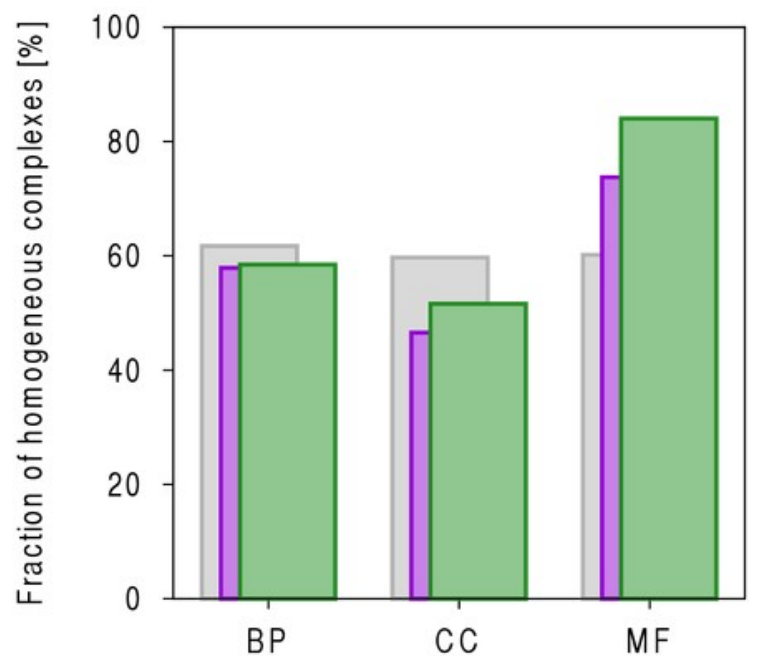




\section{Table 3 (on next page)}

Prediction of protein complexes in fly.

Basic statistics of the complex prediction results in fly, based on aligning known yeast and human complexes to the fly interactome (see Materials \& Methods). Results are shown both for all complex predictions (All) and for the high-confidence subset (HC). \#, number of; $\mathrm{nr}$, non-redundant. 


\begin{tabular}{l|c|c|c|c} 
& \multicolumn{2}{|c|}{ Yeast to fly } & \multicolumn{2}{c}{ Human to fly } \\
\hline Prediction type & All & HC & All & HC \\
\hline \# query complexes & 244 & 236 & 1027 & 645 \\
\hline \# predicted complexes (nr) & 66 & 42 & 219 & 71 \\
Total \# complex components (nr) & 405 & 255 & 640 & 291 \\
Average \# proteins per complex & 7.5 & 6.69 & 5.84 & 5.93 \\
Total \# novel components (nr) & 252 & 134 & 454 & 168 \\
Average \# novel components per complex & 4.61 & 3.55 & 3.69 & 2.79 \\
\# entirely novel complexes (nr) & 6 & 4 & 45 & 11
\end{tabular}




\section{Figure 4}

Examples for complex prediction based on network alignment.

Examples of using NetAligner [36] to predict protein complexes based on aligning network representations of known protein complexes in one species to the interactome of another species. Notably, NetAligner does not require query complexes to be fully connected. Edge colors in the alignment solutions show which species the given interaction that was predicted to be likely conserved originates from. Green edges denote known conserved interactions. High-confidence interactions are highlighted with thicker edges. Alignment solution nodes represent pairs of orthologous proteins (separated by '") between the respective species, allowing both one-to-many and many-to-many orthology relationships. Components of the given predicted complex in the target species (extracted from the respective alignment solution) are listed, together with the GO biological process and molecular function annotations that could be assigned to the predicted complex. A: prediction of a mitochondrial processing peptidase complex in human; B: prediction of a mismatch repair complex in yeast; C: prediction of a SNX complex in fly. See main text for details. Network representations were created with Cytoscape [59]. 
Network representation

of the query complex

A

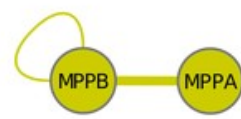

Yeast mitochondrial

processing peptidase complex

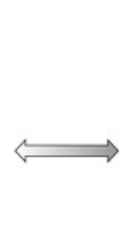

NetAligner
Target interactome

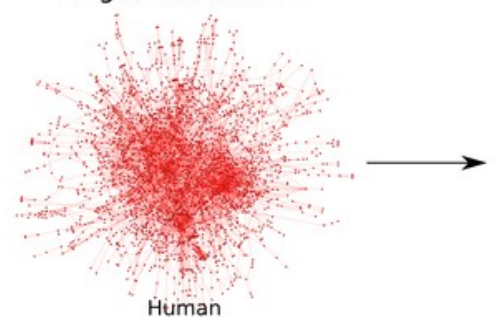

Alignment solution

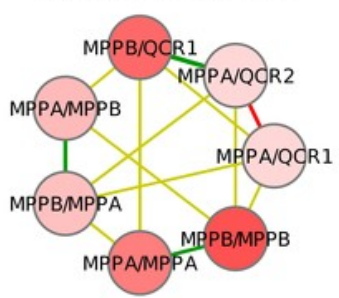

Predicted target complex

Components:

MPPA, MPPB, QCR1, QCR2

Biological process:

Proteolysis

Molecular function:

Metalloendopeptidase activity, Zinc ion binding
B

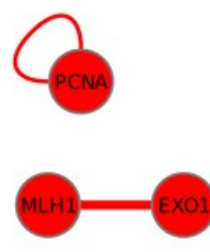

Human EXO1-MLH1-PCNA mismatch repair complex

C

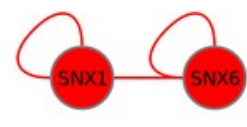

Human SNX complex
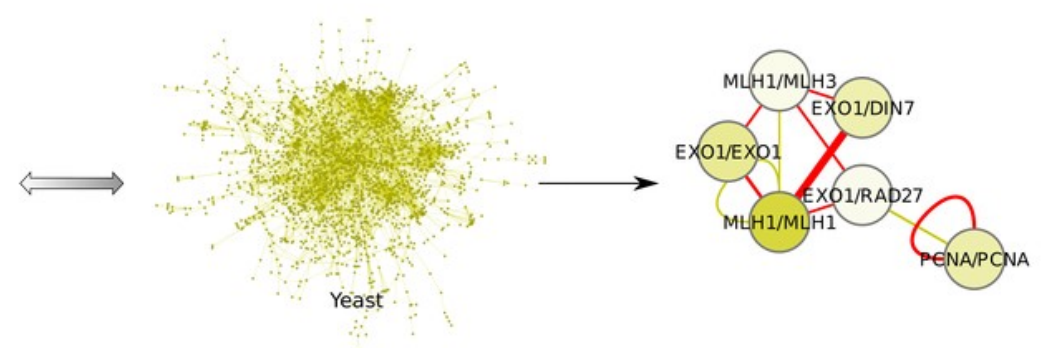

Components:

EXO1, MLH1, MLH3, DIN7, RAD27, PCNA

Biological process:

Mismatch repair

Molecular function: no common annotation

Components: Q9VLQ9, Q9VQQ6

Biological process: Cell communication

Molecular function: Phosphoinositide binding 


\section{Figure 5}

Comparison of fly complex predictions originating from yeast and human data.

Comparison of the 219 and 66 non-redundant fly complexes predicted through alignment of human (red) and yeast (yellow) query complexes, respectively, to the fly interactome using NetAligner [36]. High-confidence (HC) subsets of the complex predictions are shown in darker blue. Matching complexes, i.e. those that were identified both from human and yeast query complexes with more than $50 \%$ shared components, are highlighted in orange. The 11 pairs of matching complexes between the two HC subsets are listed below. 


\section{Human query complexes}

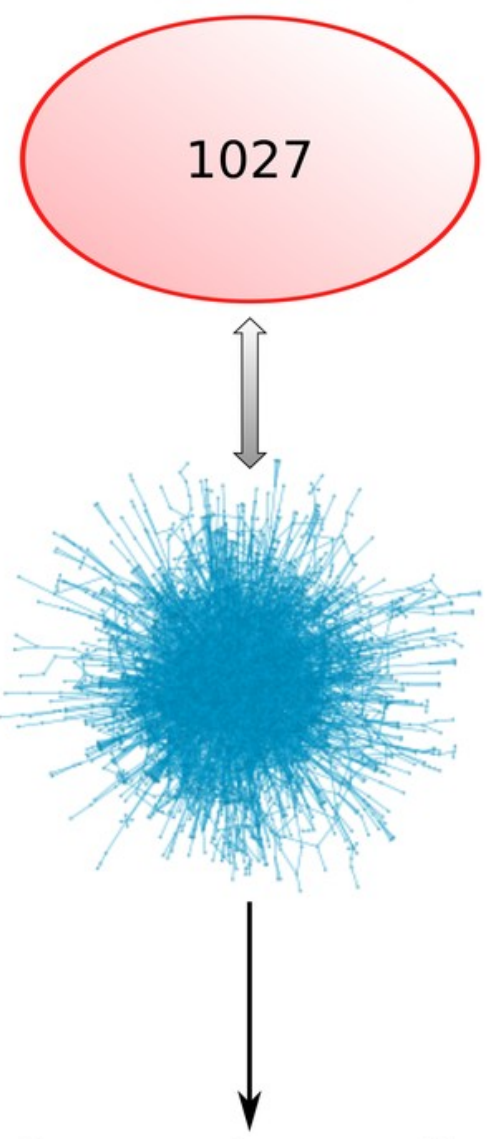

219 fly complex predictions

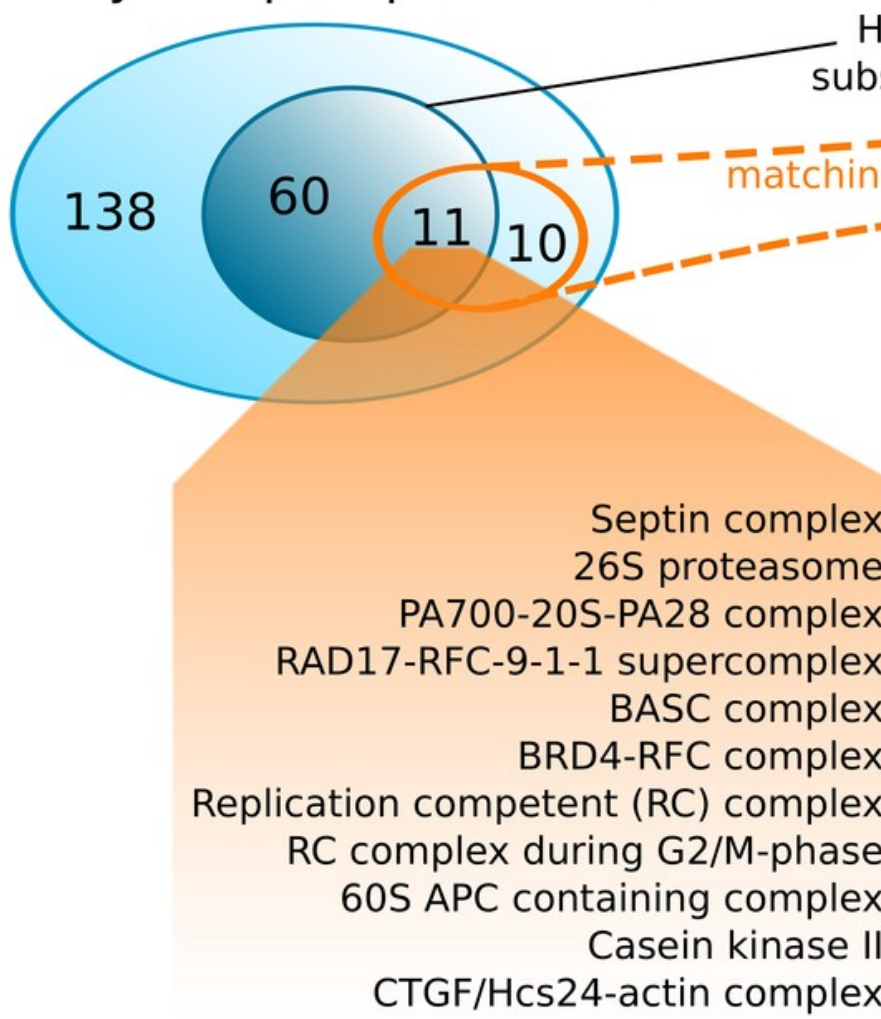

$\mathrm{HC}$

\section{Yeast query complexes}

Fly interactome NetAligner
66 fly complex predictions

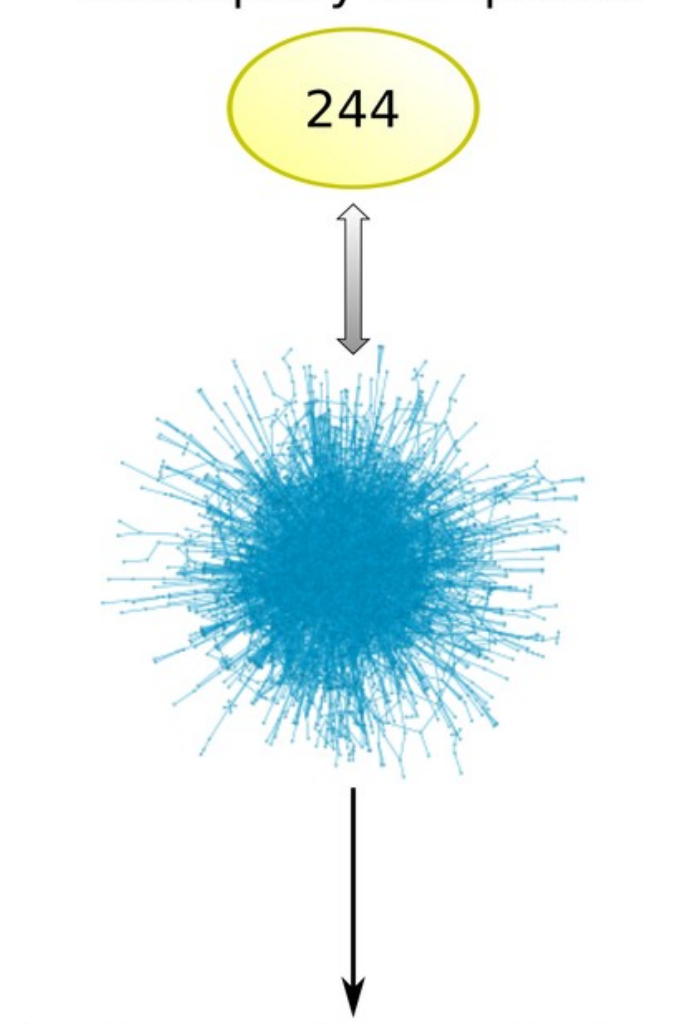
ubsets 57 35 19 complexes

\section{(1)} (exx 促 然

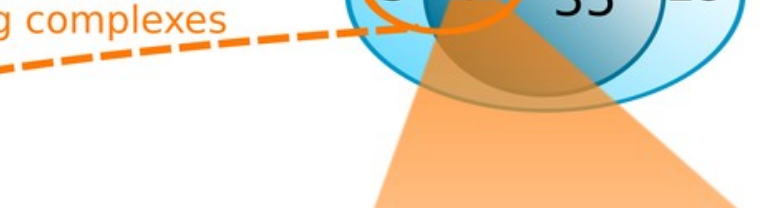




\section{Table 4 (on next page)}

Predictions based on known mycoplasma complexes.

Basic statistics of the complex prediction results in yeast, fly and human, based on aligning known mycoplasma complexes to the respective species interactome (see Materials \& Methods). Results are shown both for all complex predictions (All) and for the highconfidence subset (HC). \#, number of; nr, non-redundant. 


\begin{tabular}{l|c|c|c|c|c|c} 
& \multicolumn{9}{|c}{ Mycoplasma to } \\
& \multicolumn{2}{|c|}{ Yeast } & \multicolumn{2}{c}{ Fly } & \multicolumn{2}{c}{ Human } \\
\hline Prediction type & All & HC & All & HC & All & HC \\
\hline \# query complexes & 174 & 67 & 174 & 67 & 174 & 67 \\
\hline \# predicted complexes (nr) & 11 & 3 & 9 & 1 & 6 & 1 \\
Total \# complex components (nr) & 125 & 8 & 86 & 6 & 39 & 3 \\
Average \# components per complex & 13.64 & 2.67 & 12.0 & 6 & 7.17 & 3 \\
Total \# novel components (nr) & 86 & 1 & 68 & 4 & 30 & 0 \\
Average \# novel components per complex & 9.36 & 0.33 & 9.56 & 4 & 5.33 & 0 \\
\# entirely novel complexes (nr) & 0 & 0 & 0 & 0 & 1 & 0
\end{tabular}




\section{Figure 6}

Mycoplasma complexes leading to significant predictions in yeast, fly and human.

Comparison of the different sets of mycoplasma complexes that led to significant predictions in yeast (yellow), fly (blue) and human (red) through complex to network alignment using NetAligner [36] (grey arrows). Overlaps between the different sets are shown as a Venn diagram. Complexes found in at least two species are listed.

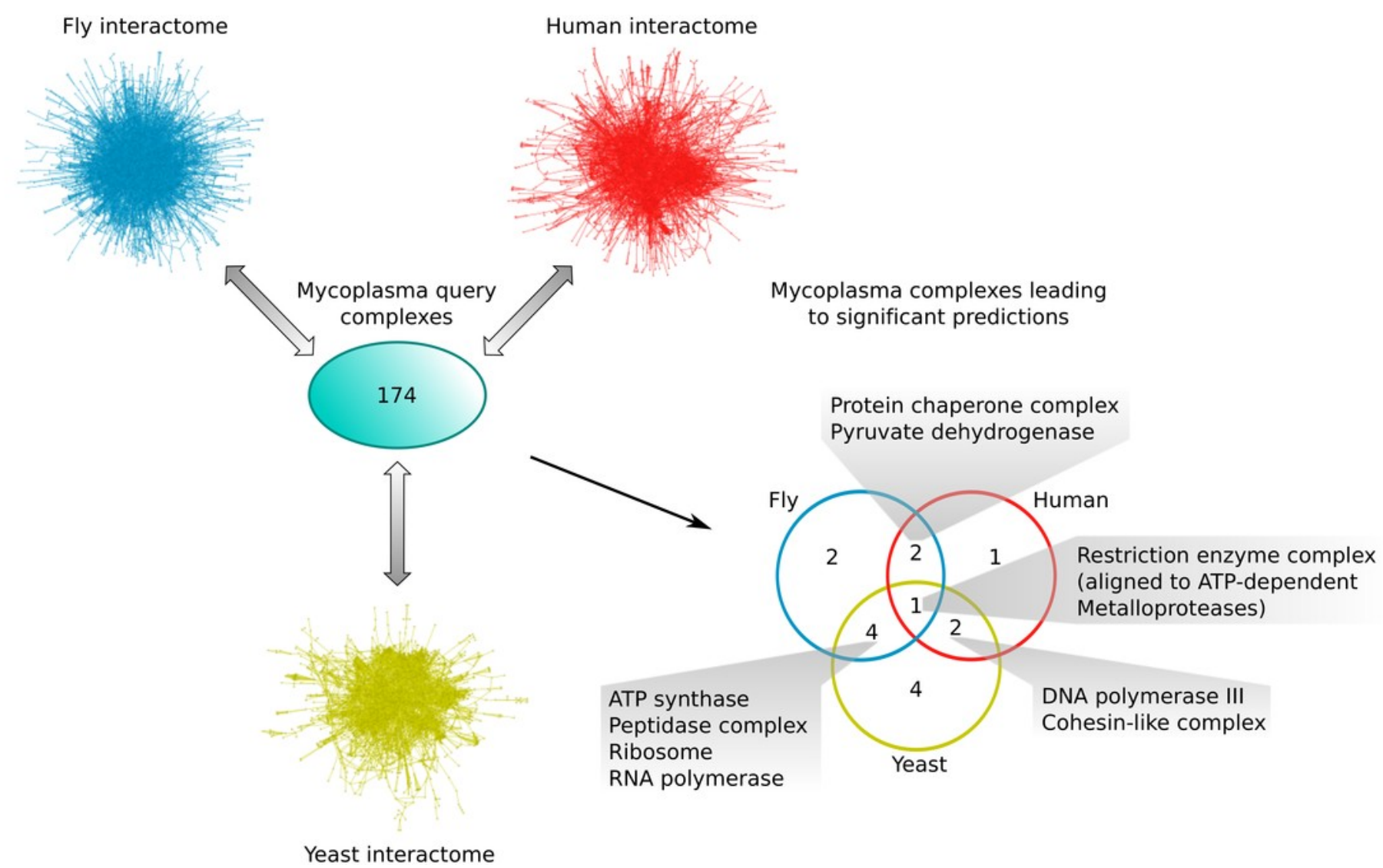

